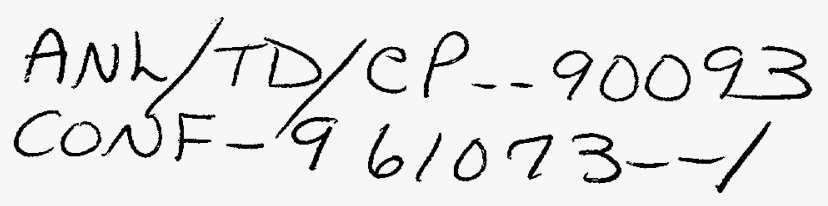

\title{
Selecting a High-Power Fiber-Optic Laser Beam Delivery System
}

\author{
BOYD V. HUNTER \\ LightPath Technologies, Inc. \\ 6820 Academy Parkway E., NE \\ Albuquerque, New Mexico 87109 \\ KENG H. LEONG \\ Argonne National Laboratory \\ Technology Development Division \\ Laser Applications Laboratory \\ 9700 South Cass Avenue, Building 207 \\ Argonne, Illinois 60439

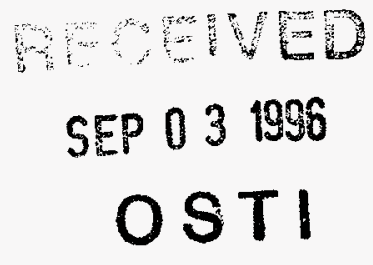 \\ U. S. Laser Corporation \\ 825 Windham Court North \\ P. O. Box 609 \\ Wyckoff, New Jersey 07481
}

Carl B. Miller, James F. Golden, Robert D. Glesias and Patrick J. LAVERTy

The submitted manuscript has been authored by a contractor of the U.S. Government under contract No. W-31-109-ENG-38. Accordingly, the U.S. Government retains a nonexclusive, royalty-free license to publish or reproduce the published form of this contribution, or allow others to do so, for U.S. Government purposes.

\section{$\underline{\text { Abstract }}$}

Multimode optical fibers enable efficient flexible laser beam delivery but at a loss in the quality of the delivered beam. The fiber-optic beam delivery system effectiveness is strengthened by the optimal selection of its components not only for minimizing beam quality degradation but also for robustness. Smaller fibers tend to produce less degradation to beam quality but the minimum usable fiber size is limited by the quality of the laser beam, focusing optic and the numerical aperture of the fiber. Selection of the appropriate fiber type is an important consideration because the characteristics of the output beam enhance or degrade the utility of the fiber-optic-delivered beam for different applications.

The other components of the beam delivery system also impact performance. High-power handling requires high-quality fiber end-face finish and special connectors that can withstand back- 


\section{DISCLAIMER}

Portions of this document may be illegible in electronic image products. Images are produced from the best available original document. 


\section{DISCLAIMER}

This report was prepared as an account of work sponsored by an agency of the United States Government. Neither the United States Government nor any agency thereof, nor any of their employees, makes any warranty, express or implied, or assumes any legal liability or responsibility for the accuracy, completeness, or usefulness of any information, apparatus, product, or process disclosed, or represents that its use would not infringe privately owned rights. Reference herein to any specific commercial product, process, or service by trade name, trademark, manufacturer, or otherwise does not necessarily constitute or imply its endorsement, recommendation, or favoring by the United States Government or any agency thereof. The views and opinions of authors expressed herein do not necessarily state or reflect those of the United States Government or any agency thereof. 
reflections. Recent developments in optical materials allow the manufacture of very low aberration optics that are particularly useful for short focal length lenses in output optics.

Harnessing the power of a high-power laser requires that knowledgeable and prudent choices be made when selecting the laser and its beam delivery system. We will focus on issues relevant to understanding and specifying a fiber-optic beam delivery system and provide some guidelines for specifying a system. Data obtained with high power Nd:YAG lasers will be used as illustrative examples.

\section{Introduction}

The widespread availability of lasers of varying power levels and wavelengths has moved laserbased material processing from a laboratory curiosity to an economically important manufacturing technology. Fiber-optic beam delivery allows the beam to be transmitted in a small, flexible cable and is ideal when the beam must be delivered along a complex path or processing requires complicated manipulation of the beam delivery optics. The flexibility associated with the fiber-optics eliminates the mechanical and optical complexities of components such as articulated joints and is well suited for use with robots.

Argonne National Laboratory's Laser Applications Laboratory and U. S. Laser Corporation have jointly investigated the performance of laser beam delivery systems and various techniques for improving the performance of the system. ${ }^{1}$ Based on the results of a series of experiments, conclusions can be drawn about the mechanisms responsible for the performance of the beam delivery system. Understanding these mechanisms enables the designer to improve the system.

This paper will investigate the parameters which influence the changes that the fiber forces on the laser beam, fiber termination and connection requirements, and the output optics. A number of innovations are possible in the output optics; a simple methodology helps quantify the performance required of the output optics.

The fiber-effect data presented in this paper were taken using a CW Nd:YAG laser (U.S. Laser, $2 \mathrm{~kW}$ ). The beam was launched into a series of fibers of different lengths, core sizes and refractive index profiles. The output optics profiles were taken using a pulsed Nd:YAG laser (Electrox, 1.6 $\mathrm{kW}$ ). The beam profiles were obtained using a beam analyzer (Prometec Laserscope).

\section{Effect of the fiber on the beam}

\section{Fiber type}

The optical fiber is a fundamentally quantized device capable of supporting only a discrete set of modes. These discrete modes, or eigenmodes, are determined uniquely by the fiber refractive index profile and geometry and can often be determined analytically. ${ }^{2,3,4}$ Fiber modes can be separated into two general classes: radial and azimuthal (also called meridional and skew, respectively). Radial modes always go through the fiber's axis. Azimuthal modes do not pass through the fiber's axis; the rays propagate in a helical path. Unsupported modes exceed a specific 
maximum propagation angle and will either leak out of the fiber or be canceled/redistributed due to interference effects. A supported mode may be subjected to losses because of scattering from surface or inhomogeneities in the material. Introducing bends in the fiber locally perturbs the supported mode structure and may cause local mode mixing and losses. The fiber is capable of significantly modifying the properties of the laser beam; the final processing properties of a fiberoptic-delivered laser beam are determined by the fiber type, length, and propagation conditions.

The two fiber types differ in the constancy of the refractive index through the fiber. The commonly used step-index fiber has a constant refractive index in the core and an abrupt "step" transition to a different

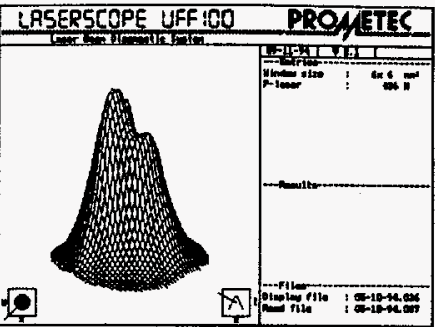

(a) $25 \mathrm{~mm}$ from fiber face.

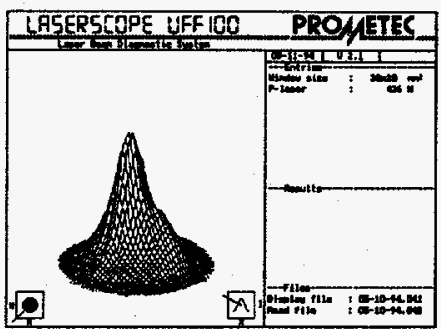

(c) $75 \mathrm{~mm}$ from fiber face.

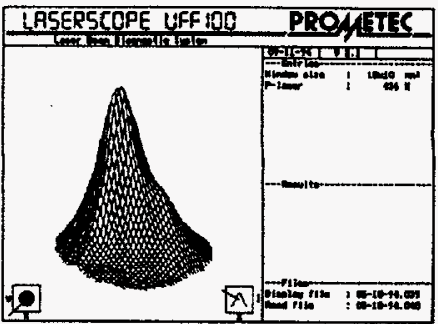

(b) $50 \mathrm{~mm}$ from fiber face.

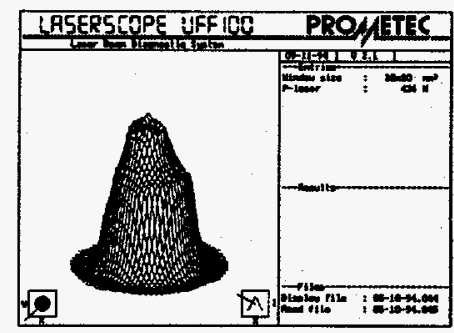

(d) Collimated beam from fiber.

Figure 1. Profiles of the evolving beam profile from an $800 \mu \mathrm{m}$ step-index fiber (2) 25 , (b) 50 and (c) 75 $\mathrm{mm}$ from the fiber face. The profile of the (d) collimated beam emphasizes azimuthal modes by making the crescent more visible. internal reflection in this fiber occurs at the core/cladding interface for all modes. When all modes are filled, the output profile (on the fiber face) will be the familiar "top-hat" distribution.

A gradient-index (also called graded-index) fiber has a variable refractive index in the core. Typically, the variation of the refractive index is a parabolic function of the radius. The effect of this varying refractive index profile is that each mode is refracted gradually as it traverses the fiber. Each mode, therefore, has a unique mode radius. Except for the evanescent components, only the highest order mode reaches the core/cladding interface. The output profile (on the fiber face) resembles a Gaussian distribution, although, for the same fiber diameter, the $86 \%$ radius is smaller. The peak intensity is often about five times higher than a step-index fiber.

The transformation of the laser beam as it propagates through the fiber is evident not only on the fiber face (reimaged at the beam focus), but also as it propagates out of the fiber. After exiting the fiber, a wide variety of intensity distributions are possible because of the large number of modes present. Figure 1 shows the evolving profiles of the diverging beam from a $2 \mathrm{~m}$ length of $800 \mu \mathrm{m}$ step-index fiber. These diverging profiles quickly take on a quasi-Gaussian shape.

These output profiles can be used to optimize the launching of the beam. Misalignment of the fiber at the launch end preferentially induces azimuthal modes that appear as rings or crescents in the output. Typically, for step-index fibers, these rings are found near the periphery of the fiber. ${ }^{5}$ The "shoulder" in the profiles in Figure 1 is indicative of a slight misalignment of the fiber and launch optics. Collimating this output beam (Figure 1d) makes the contributions from the higher angular momentum modes appear more distinctly as a crescent or ring. Figure 2 shows the focused 
spot profiles from a $3 \mathrm{~m}$ length of $800 \mu \mathrm{m}$ gradient-index fiber when properly aligned and severely misaligned. These profiles dramatically illustrate the shift to azimuthal modes because of misalignment; the ring structure is so dominant that the Gaussian shape now resembles a D-mode.

\section{Fiber length}

The light launched into the fiber has some initial distribution determined by the laser. As the light propagates in the fiber, it is converted into modes supported by the fiber or it is lost and appears as leakage or heat. This conversion, however, requires a certain length of fiber to be effected. There are different length scales that must be considered: short, intermediate, and long.

A short fiber produces little modification to the input beam. This case is not of practical significance because the fiber is essentially a window. On the other hand, a long fiber causes the output to be independent of the input distribution.

Fiber lengths used in many applications fall into the intermediate category. The length scale of "intermediate" depends upon many factors, including launch conditions, input distribution, fiber inhomogeneities, and bends. ${ }^{6,7,8}$ Some feel for the relevant length scale comes from the fact that if the launching optics focus the light into a spot much smaller than the fiber core, it will take $10 \mathrm{~m}$ to $1000 \mathrm{~m}$ to reach steady-state. ${ }^{9}$ This transformation of the input by intermediate length fibers is illustrated with "before" and "after" beam intensity profiles taken with a $436 \mathrm{~W}$ CW Nd:YAG beam delivered through the $800 \mu \mathrm{m}$ step-index fiber. Figure 3 shows the focused beam launched into the fiber (Figure 3a) and the focused output from the fiber (Figure $3 b$ ). The distinctive double peak structure of the input beam is a common signature of the pumping method used in this laser. The fiber has significantly

(a) Focused beam launched into the fiber.

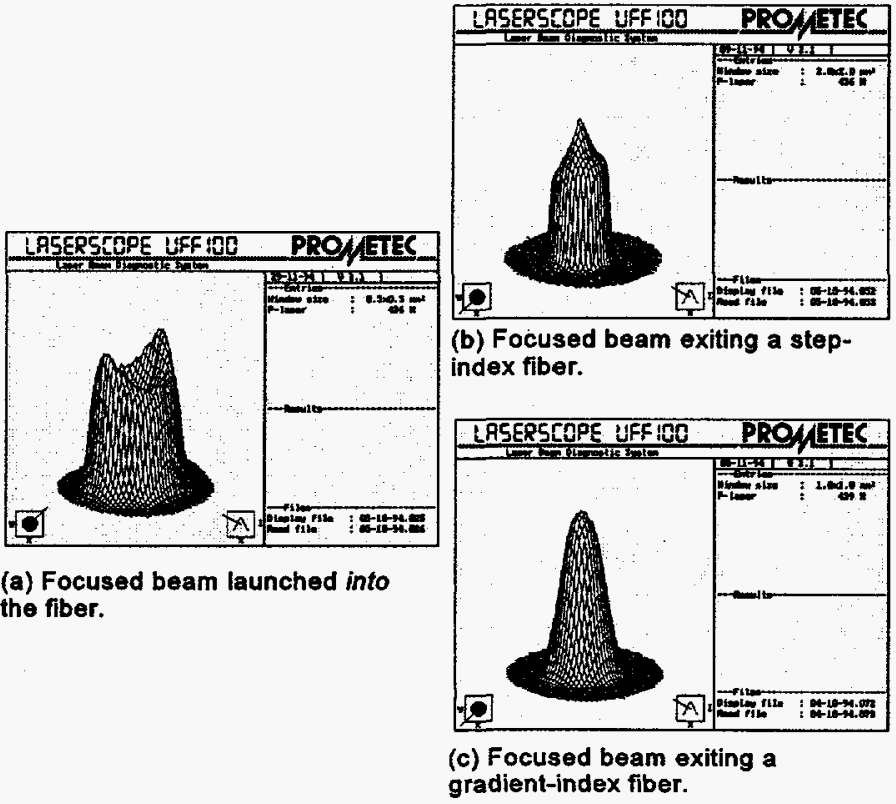

Figure 3. Focused beam profiles for the $436-439 \mathrm{~W}$ Nd:YAG laser beam (a) launched into the fibers and exiting the (b) step-index and (c) gradient-index 800 $\mu \mathrm{m}$ fibers.

altered the beam profile and masks many properties of the source laser. The focused beam profile is, subject to aberrations and magnification effects of the imaging lens, the profile on the fiber output face. The intermediate length of the fiber was not long enough to allow the formation of the top-hat profile expected for a long step-index fiber. Instead, a pointed top profile was produced. This type 
of profile was typical of output obtained from several $\mathrm{CW}$ multikilowatt Nd:YAG lasers (different manufacturers) using $1000 \mu \mathrm{m}$ fibers of $10 \mathrm{~m}$ length.

\section{Fiber core size}

Fiber size is an important consideration in deploying a beam delivery system. Smaller fibers produce less degradation of beam quality. Using a smaller fiber (assuming the same numerical aperture) allows the same focused spot size to be achieved with a greater stand-off distance or, alternatively, smaller spot sizes to be achieved. The smaller spot sizes result in higher irradiances; many applications require high irradiances that can only be achieved by maintaining beam quality when power is limited. The benefits expected from smaller fibers are important because multikilowatt Nd:YAG systems often have their usefulness limited by available power.

The worst case beam quality from a step-index fiber can be estimated using the conventional definition of $M^{2}$. This definition is based on a Gaussian beam and the $86 \%$ energy enclosure beam waist is used. If we substitute $0.86^{1 / 2} r_{\text {fiber }}$ for the beam waist and make the necessary adjustment for the angle of divergence using $0.86^{1 / 2} \arcsin (\mathrm{NA})$, then

$$
M^{2}=0.86 r_{f i b e r} \arcsin (N A) \frac{\pi}{\lambda}
$$

The worst-case estimate for a gradient-index fiber is considerably more difficult to make. However, the beam quality will be better than the step-index case.

There are important limitations to selecting the appropriate (smallest) fiber size. Clearly, smaller fibers are preferred in order to get the best beam quality. However, the laser itself imposes constraints on the smallest fiber that can be used. The laser beam focused spot size has to be smaller than the fiber core to avoid heat effects and allow for the mechanical tolerances of the fiber-optic connectors. The laser's beam quality and the numerical aperture (NA) of the fiber constrain the focal length of the launch optics. Working from the equation for the focused spot size for an $\mathrm{M}^{2}$-timesdiffraction-limited beam, given the ratio of $86 \%$-to- $100 \%$ radii in the collimated beam exiting the laser is $a$, the ratio in the focused beam on the fiber face is $b$, and the desired fiber fill factor is $c$, the minimum fiber diameter, $d$, to be selected as a function of $\mathrm{M}^{2}, \mathrm{~K}, \lambda$ and the NA is given by

$$
d=M^{2} K \frac{2 a b}{c} \frac{\lambda}{\pi \tan (\arcsin [N A])}
$$

where $\mathrm{K}$ is the aberration of the optics and $\lambda$ is the wavelength. If $a$ and $b$ are 1.5 (based on our experience) and in the interest of conservative engineering, $c$ is 0.8 , at a wavelength of $1.064 \mu \mathrm{m}$, $\mathrm{NA}=0.2$ and $\mathrm{M}^{2} \mathrm{~K}=70$, the minimum fiber diameter would be $650 \mu \mathrm{m}$. If $\mathrm{M}^{2} \mathrm{~K}=100$, then the size increases to $930 \mu \mathrm{m}$. Consequently, most high power Nd:YAG laser systems use 800 or $1000 \mu \mathrm{m}$ fibers for beam delivery. However, if the performance of the laser (ratio of the $86 \%$ and $100 \%$ radii) is better than given by these conservative assumptions or a larger fill factor is used, the beam can be launched reliably into smaller fibers. As an example, at U.S. Laser with $\mathrm{M}^{2} \mathrm{~K} \sim 80$, the $2 \mathrm{~kW} \mathrm{CW}$ laser beam is routinely launched into $560 \mu \mathrm{m}$ fibers. 


\section{Fiber surface finish}

The primary damage mechanism for fiber-delivered $\mathrm{CW}$ lasers in the $\mathrm{kW}$ power regime is heating in the connectors. If the fiber's input and output optics are properly designed, most of the connector heating comes from spurious reflections or refractions at the fiber end faces. The primary causes are the refractive index discontinuity at the fiber surface (Fresnel losses) and scattering from surface imperfections. The Fresnel losses can potentially be minimized by applying antireflection coatings to the fiber. The scattering can be reduced by optimizing the surface quality. In both cases, the fiber finishing technique is very important. It not only reduces the scattering losses but also affects the quality and adhesion of AR coatings.

We had several fiber samples prepared and sent to an independent testing laboratory for surface evaluation. The samples were prepared using three different techniques: mechanical polish, cleaving and laser polishing. In addition, mechanical polishing was done by three different vendors to evaluate their respective capabilities. The sample fiber surfaces were evaluated with a Wyko interferometer. They were tested for RMS roughness and peak-to-valley surface figure

\begin{tabular}{|c|c|c|c||}
\hline Technique & & $\begin{array}{c}\text { RMS Surface } \\
\text { Roughness } \\
(\mathbf{n m})\end{array}$ & $\begin{array}{c}\text { Peak-to- } \\
\text { Valley Surface } \\
\text { Error }(\mu \mathbf{m})\end{array}$ \\
\hline \multirow{3}{*}{$\begin{array}{c}\text { Mechanical } \\
\text { Polish }\end{array}$} & Vendor A & 0.4 & 3.3 \\
\cline { 2 - 4 } & Vendor B & 1.5 & 3.3 \\
\cline { 2 - 4 } & Vendor C & 1.7 & 4.1 \\
\hline Cleave & & wavy & 2.5 \\
\hline Laser Polish & & 0.3 & 4.1 \\
\hline
\end{tabular}
error (deviation from a perfectly flat surface). The RMS roughness gives an indicator of microscopic roughness; the peak-to-valley surface figure error is an indicator of the macroscopic surface flatness. Both mechanical and laser polishing resulted in slightly aspheric convex fiber end faces. The results for $1000 \mu \mathrm{m}$ core fibers are presented in Table 1. From this data, it appears that the high quality mechanical polish can provide both local smoothness and overall flatness for the optimum finish. The mechanical polish quality varied significantly among vendors $\mathrm{A}, \mathrm{B}$, and $\mathrm{C}$. These variations are a direct function of each vendor's polishing techniques. Since polishing techniques are proprietary and are somewhat of an art, care must be taken to select vendors who can meets strict specifications.

Laser polishing is a technique that uses a $\mathrm{CO}_{2}$ laser to melt the fiber end, yielding a smooth finish after cooling and solidification. Laser polishing achieved the best overall RMS roughness (local smoothness) but yielded larger peak-to-valley values than the best mechanical polish. The laser polished ends were observed to take on a quasi-spherical curvature, protruding toward the middle of the fiber and receding near the edges. This is most likely due to surface tension effects during 
solidification. The fiber end curvature may be enough to cause focusing effects and degrade fiber-tolaser coupling. Overall, it was determined that laser polishing provides no particular advantages that cannot be achieved through less costly means.

Besides mechanical and laser polish, several fiber samples were cleaved and evaluated. Cleaving has traditionally been considered a superior method of end finish because the induced break generally provides a smooth, flat finish. Little work had been done, however, in cleaving large core multimode fibers for high power beam delivery. The vendor we used had recently developed a proprietary fixture and methodology for cleaving such fibers. The samples evaluated showed good local smoothness, but had large scale ripples or waves that made it impossible to get reliable interferometer readings. In addition to waviness, the cleaving process can impart a burr near the fiber edge, which could lead to disastrous consequences in extreme high power usage. We found no vendors who could provide a repeatable cleave that was as good as a mechanical polish.

Properly finished fibers, together with good cleaning techniques, provide a surface to which AR coatings can adhere well. U. S. Laser has tested several AR coatings from different vendors and has found a direct correlation between the surface polish quality and the quality of the coating. With some effort, it is possible to get reliable, durable coatings. U. S. Laser has combined AR-coated fibers with proprietary injection optics to achieve $>\mathbf{9 8 \%}$ total transmission efficiency through the fiber-optic beam delivery system, including both injection and output collimation optics.

\section{Connectors}

A mechanically robust connector should perform well for repeatable precision reconnections and be rugged. However, high power applications impose stricter requirements on connector designs. For the beam input end, if the beam is launched properly with no undue heat effects, standard fiberoptic connectors can be effectively used. For example, the Electrox $1.6 \mathrm{~kW}$ pulsed Nd:YAG laser at Argonne National Laboratory has used a standard ST connector bored out for a $1 \mathrm{~mm}$ fiber core. On the other hand, the output end of the fiber is subject to more severe conditions with reflections from the workpiece in addition to frequent mechanical manipulation. The reflected laser energy incident on the epoxy, commonly used to hold the fiber core in the connector, may cause melting and vaporization that will affect the beam propagation out of the fiber end. The solution found in high power connectors is to use a well type ferrule connection that holds the fiber tip in air. The ferrule mass is also increased for improved thermal loads. Frequently, the last 20 to $30 \mathrm{~cm}$ length of the output end of the fiber is sheathed in a rigid casing to allow for handling ruggedness and to prevent bending of the fiber and maintain beam quality.

\section{Output optics}

The laser beam delivered by the fiber needs to be transformed for a particular application. The conventional technique is to upcollimate and focus to a small spot. The collimating optic speed is dictated by the NA of the fiber. Depending on how the beam is launched into the fiber, the actual fiber NA may be less than the rated (maximum) NA. With large core fibers, short focal length optics are often needed to meet high irradiance requirements. Cover glass protection is essential with the short standoffs and susceptibility to spatter. 
Because of the large number of modes supported by the fibers used for high-power beam delivery, diffraction effects are minimal; geometrical image analysis is appropriate. The optical system images the fiber face on to the workpiece. Diffraction and aberrations increase the actual spot size from this geometrical baseline by blurring the image of each point on the fiber face. Geometrically, the size of the image of the fiber face is given by

$$
r=m \cdot r_{\text {fiber }}+r_{\text {blur }}
$$

where $m$ is the absolute value of the magnification of the imaging system, $r_{f i b e r}$ is the fiber radius and $r_{\text {blur }}$ is the blur introduced because of diffraction effects or aberrations in the optical system. The magnification is determined by the design of the optical system. The importance of the blur cannot be ignored.

Figure 4 shows focused beam profiles from a $1 \mathrm{~mm}$ step-index fiber imaged with two similar optical systems $(m=0.5)$. One system used standard, off-the-shelf BK7 plano-convex singlets, which introduced significant aberration. The second system used singlets made from GRADIUM ${ }^{\mathrm{TM}}$, a material with an axial refractive index gradient. The GRADIUM material allows highly-corrected (nearly aberration-free) lenses to be manufactured using standard spherical surfaces. The improvement is very clear. Not only does

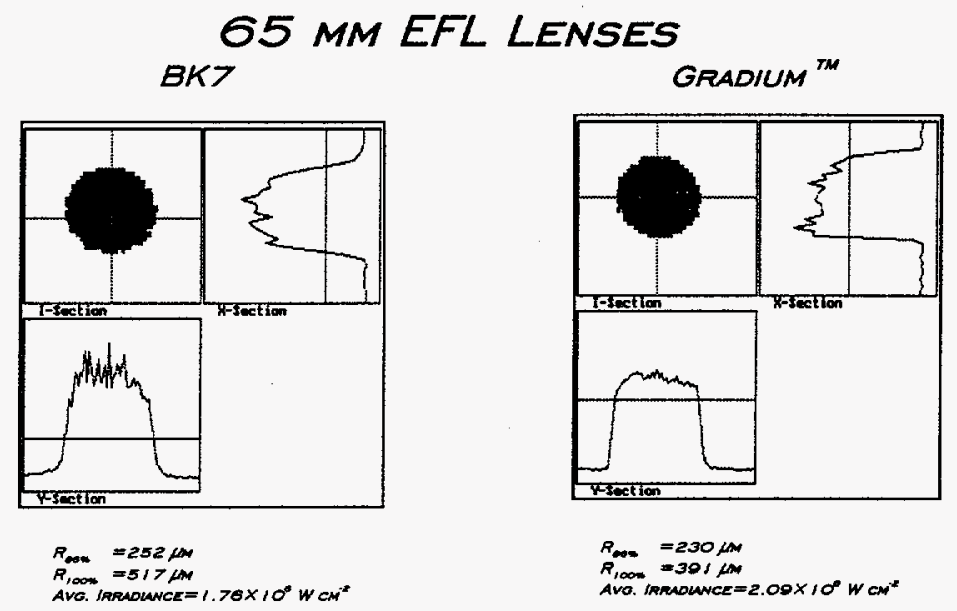
the GRADIUM nearly eliminate the blur due to aberrations, but it also reduces the shift in focal position as the laser power changes (and the mode structure out of the fiber changes) to insignificant levels. The same result could also be obtained with well-corrected doublets, triplets, aspherics, or conventional/diffractive combinations. Starting from equation (3), the same rationale used to determine depth of focus ( $5 \%$ increase) can be used to determine the maximum aberration which will result in no significant increase in spot size: $r_{b l u r}=0.05 \mathrm{~m} \cdot r_{\text {fiber }}$. This maximum aberration provides a design target for the geometrical spot size that can be used with commercial ray-tracing software. Any optical system that performs at or below this level will be effectively indistinguishable from a perfect, diffraction-limited system. Because of the apodization created by the beam exiting the fiber and detector thresholds, the actual detection limit will allow even more aberration than indicated by this argument. Our data indicate that the $r_{86}$ size changes less than $5 \%$ if the ray-trace rms blur is 
$s 0.25 m \cdot r_{\text {fiber }}$ Larger rms blur values may result in more than $5 \%$ increase, depending upon the details of the optical configuration.

\section{Application considerations}

The importance of the choices made in a beam delivery system is illustrated by considering the power levels required for welding steel and aluminum, processes with an irradiance threshold. The laser beam power required to exceed the threshold irradiance can be computed by

$$
P_{t}=0.86 \frac{\pi I_{t}}{T}\left(\frac{w_{r}}{F}\right)^{2}
$$

where $I_{t}$ is the threshold irradiance, $w_{r}$ is the focused $86 \%$ energy enclosure radius, $\mathrm{T}$ is the power transmission and $\mathrm{F}$ is a factor that depends on the characteristic of the fiber. We define $F=1$ for step-index fibers; $F$ is greater than 1 for gradient-index fibers resulting in a substantial decrease in the threshold power requirement. The requirements for welding are indicated in Table 2 for steel and 5000 series aluminum alloys. Table 2 shows that reducing the size of $w_{r}$ (either by using a smaller core diameter fiber, gradient-index fiber, or shorter focal length lenses) significantly reduces the total laser power required.

\begin{tabular}{|c|c|c|c|}
\hline \multirow{2}{*}{ Fiber Type } & \multirow{2}{*}{$\mathbf{W}_{\mathbf{r}}(\mu \mathbf{m})$} & \multicolumn{2}{|c|}{$\begin{array}{c}\text { Threshold Laser } \\
\text { Beam Power (W) }\end{array}$} \\
\cline { 3 - 4 } & & steel & aluminum \\
\hline \multirow{2}{*}{ Step index } & 300 & 860 & 2900 \\
\cline { 2 - 4 } & 200 & 380 & 1300 \\
\hline \multirow{2}{*}{ Gradient index } & 210 & 440 & 1500 \\
\cline { 2 - 4 } & 140 & 190 & 620 \\
\hline
\end{tabular}

Table 2. Threshold laser beam power required for welding steel and aluminum using $800 \mu \mathrm{m}$ fibers. Threshold irradiances of $3 \times 10^{5}$ and $10^{6} \mathrm{~W} \cdot \mathrm{cm}^{-2}$ were assumed for steel and aluminum respectively. $85 \%$ transmission was assumed. The $86 \%$ beam radii used correspond to focal lengths of 65 to $100 \mathrm{~mm}$.

\section{Conclusions}

Multimode optical fibers enable efficient flexible laser beam delivery but at a loss in the quality of the delivered beam. The fiber-optic beam delivery system effectiveness is strengthened by the optimal selection of its components not only for minimizing beam quality degradation but also for robustness. Some of the key issues to be addressed when selecting a beam-delivery system are:

- The laser beam to be delivered should be launched properly into the fiber, otherwise damage may arise. Coarse alignment of the beam launch conditions is carried out by checking transmitted power. Fine alignment can be obtained by monitoring the beam profile exiting the fiber for azimuthal modes. 
- Smaller fibers tend to produce less degradation to beam quality but the spot size of the beam focused into the fiber is limited by the quality of the laser beam, focusing optic and the numerical aperture of the fiber. The fiber type strongly influences the focused spot size on the workpiece. - Highly-corrected optics can significantly improve the performance of the beam-delivery system. Aberration tolerances can be easily estimated given the desired size of the imaged fiber face.

- High power handling requires special connectors that can withstand back-reflections and fibers with high-quality surface finishes.

\section{$\underline{\text { References }}$}

1. Leong, K. H. and B. V. Hunter (May, 1996). High-power fiberoptic laser beam delivery moves forward. Industrial Laser Review 11: 7-12.

2. Jackson, J. D. (1975). Classical Electrodynamics. 2nd ed. Wiley, New York. 109.

3. Lee, D. L. (1986). Electromagnetic Principles of Integrated Optics. Wiley, New York. 288298.

4. März, R. (1995). Integrated Optics: Design and Modeling. Artech House, Boston. 69-73.

5. Hunter, B. V. and W. S. Bickel (1995). Geometry-induced fluorescence resonances in small lossy capillaries. Applied Optics 34: 4240-4247.

6. Beck, T., N. Reng and K. Richter (October, 1993). Fiber type and quality dictate beam delivery characteristics. Laser Focus World 29: 111-115.

7. Boechat, A. A. P., D. Su and J. D. C. Jones (1993). Dependence of output near-field beam profile on launching conditions in graded-index fibers used in delivery systems for Nd:YAG lasers. Applied Optics 32: 291-297.

8. Su, D., A. A. P. Boechat and J. D. C. Jones (1992). Beam delivery in large core fibers: effect of launching conditions on near-field output profile. Applied Optics 31: 5816-5821.

9. Brown, T. G. (1995). Optical Fibers and Fiber-Optic Communications in Handbook of Optics. Vol. II. M. Bass, editor-in-chief. McGraw-Hill: New York. 10.13.

\section{Meet the Authors}

Boyd Hunter is the manager of New Product Development and Technical Services at LightPath Technologies, the manufacturer of GRADIUM. When this work was performed, he was a member of the Laser Applications Laboratory (LAL). Keng Leong is the manager of the LAL at Argonne National Laboratory. Recent LAL projects include improved beam delivery systems, weld monitoring (patent pending), and laser welding of aluminum and vanadium alloys. Carl Miller, James Golden, Robert Glesias and Patrick Laverty are from U.S. Laser Corporation, a manufacturer of industrial Nd:YAG lasers and laser systems for various materials processing applications. 